\title{
Hipotiroidismo en pacientes del Centro de Especialidades Médicas; IESS-La Libertad
}

\author{
Hypothyroidism in patients from the Center for Medical Specialties; IESS- Libertad \\ Hipotireoidismo em pacientes do Centro de Especialidade Médicas; IESS-Libertad
}

\author{
Aleyda Ponce Loor \\ aleyda.ponce.19@est.ucacue.edu.ec \\ lelitacuchi@hotmail.com \\ https://orcid.org/0000-0001-7857-6702

\begin{abstract}
Centro de Especialidades IESS La Libertad, Cuenca-Ecuador
Programa de Maestría en Diagnóstico de Laboratorio Clínico y Molecular Universidad Católica de Cuenca, Cuenca-Ecuador
\end{abstract}

Recibido 2 de marzo 2021 | Arbitrado y aceptado 26 de marzo 2021 | Publicado en 4 de mayo 2021

\begin{abstract}
RESUMEN
El hipotiroidismo es el cuadro clínico resultante de la disminución en la producción de hormonas tiroideas. Objetivo. Fue caracterizar el hipotiroidismo en pacientes que acudieron al laboratorio clínico del Centro de Especialidades IESS de la Libertad, durante el período enero 2018 - enero 2020 mediante la medición de niveles de FT3, FT4, TSH. Materiales y Métodos. Se realizó un estudio de tipo descriptivo, de diseño documental. El universo constituye 2451 pacientes con sospecha de enfermedad tiroidea que acudieron al servicio de laboratorio del Centro de Especialidades IESS, La Libertad durante el período enero 2018 a enero 2020. Se procedió a revisar archivos de historial de resultados de los pacientes atendidos en el laboratorio. Resultados. 617 pacientes cumplen criterios de hipotiroidismo primario lo cual representa una prevalencia del 25,2\%. El hipotiroidismo subclínico, constituye el 70,8\%, el hipotiroidismo clínico representa el 29,2\%, estos fueron más frecuentes en mujeres. En cuanto al grupo etario el hipotiroidismo subclínico se presenta con mayor frecuencia en el grupo de adultos 44,9 \% y en adultos jóvenes 24,8 \%. En referencia a las comorbilidades asociadas; tanto en el hipotiroidismo clínico y subclínico las más representativas en orden de frecuencia son: hipertensión arterial (35\%), dislipidemia $(25,8 \%)$ y diabetes mellitus $(14,1 \%)$. Conclusiones. Existe una alta prevalencia de hipotiroidismo primario, los hombres se clasifican mayoritariamente con hipotiroidismo clínico, las mujeres tienen mayor frecuencia de hipotiroidismo subclínico. La presencia de comorbilidades como hipertensión arterial, dislipidemias y diabetes mellitus tipo II están presentes en los pacientes hipotiroideos estudiados.
\end{abstract}

Palabras clave: Hipotiroidismo subclínico; hipotiroidismo clínico; TSH; FT4; Comorbilidades
AP: Licenciada en Laboratorio Clínico Universidad Laica Eloy Alfar o de Manabí. Postgradista Universidad Católica de Cuenca. Maestría en Diagnóstico de Laboratorio y Molecular. Universidad Católica de Cuenca, Ecuador.

\begin{abstract}
Hypothyroidism is the clinical picture resulting from decreased thyroid hormone production. Objective. Was to characterize hypothyroidism in patients attending the clinical laboratory of the Centro de Especialidades IESS de la Libertad, during the period January 2018 - January 2020 by measuring levels of FT3, FT4, TSH. Materials and Methods. A descriptive study of documentary design was performed. The universe constituted 2451 patients with suspected thyroid disease who attended the laboratory service of the Centro de Especialidades IESS, La Libertad during the period January 2018 to January 2020. We proceeded to review the results history files of the patients attended in the laboratory. Results. 617 patients met criteria for primary hypothyroidism which represents a prevalence of $25.2 \%$. Subclinical hypothyroidism constitutes $70.8 \%$, clinical hypothyroidism represents $29.2 \%$, these were more frequent in women. Regarding the age group, subclinical hypothyroidism was more frequent in adults $44.9 \%$ and in young adults $24.8 \%$. In reference to the associated comorbidities; both in clinical and subclinical hypothyroidism the most representative in order of frequency are: arterial hypertension (35\%), dyslipidemia (25.8\%) and diabetes mellitus (14.1\%). Conclusions. There is a high prevalence of primary hypothyroidism, men are mostly classified with clinical hypothyroidism, women have a higher frequency of subclinical hypothyroidism. The presence of comorbidities such as arterial hypertension, dyslipidemias and type II diabetes mellitus are present in the hypothyroid patients studied.
\end{abstract}

Key words: Subclinical hypothyroidism; clinical hypothyroidism; TSH; FT4; Comorbidities 
AP: Licenciada en Laboratorio Clínico Universidad Laica Eloy Alfar o de Manabí. Postgradista Universidad Católica de Cuenca. Maestría en Diagnóstico de Laboratorio y Molecular. Universidad Católica de Cuenca, Ecuador.

\begin{abstract}
RESUMO
O hipotireoidismo é o quadro clínico resultante da diminuição da produção de hormônios da tireóide. Objetivo. Caracterizar o hipotireoidismo em pacientes que freqüentam o laboratório clínico do Centro de Especialidades IESS de la Libertad, durante o período de janeiro de 2018 a janeiro de 2020, medindo os níveis de FT3, FT4, TSH. Materiais e métodos. Foi realizado um estudo descritivo do projeto documental. 0 universo consistia de 2451 pacientes com suspeita de doença da tireóide que compareceram ao serviço laboratorial do Centro de Especialidades IESS, La Libertad, durante o período de janeiro de 2018 a janeiro de 2020. Procedemos à revisão dos arquivos de histórico dos resultados dos pacientes atendidos no laboratório. Resultados. 617 pacientes preencheram os critérios para o hipotireoidismo primário, o que representa uma prevalência de 25,2\%. 0 hipotireoidismo subclínico constituía 70,8\%, o hipotireoidismo clínico $29,2 \%$, e estes eram mais frequentes nas mulheres. Em termos de faixa etária, o hipotireoidismo subclínico foi mais freqüente em adultos $(44,9 \%)$ e em adultos jovens $(24,8 \%)$. Em referência às co-morbidades associadas; tanto no hipotireoidismo clínico como no subclínico, as mais representativas em ordem de frequência são: hipertensão arterial (35\%), dislipidemia $(25,8 \%)$ e diabetes mellitus $(14,1 \%)$. Conclusões. Há uma alta prevalência de hipotireoidismo primário, os homens são na maioria classificados com hipotireoidismo clínico, as mulheres têm uma maior frequência de hipotireoidismo subclínico. A presença de co-morbidades como hipertensão, dislipidemia e diabetes mellitus tipo II estão presentes nos pacientes hipotireóides estudados.
\end{abstract}

Palavras-chave: Hipotireoidismo subclínico; hipotireoidismo clínico; TSH; FT4; Comorbidades

\section{INTRODUCCIÓN}

$\mathrm{L}$ as enfermedades de la glándula tiroides, excluyendo el nódulo tiroideo y el cáncer de tiroides, constituye una de las patologías más habituales en la visita al Endocrinólogo (1). Los desórdenes de la tiroides se pueden dividir en dos grupos generales, los relacionados con la producción de hormonas tiroideas, tales como, hipotiroidismo e hipertiroidismo que son los más representativos estadísticamente y aquellos con compromiso estructural de la glándula, como el bocio y las neoplasias (2). El hipotiroidismo, se produce por disminución en la producción de hormonas tiroideas. El diagnóstico clínico se realiza mediante interrogatorio, examen físico y humoral (3). Cuando el hipotiroidismo se produce por lesión de la glándula tiroidea, se conoce como hipotiroidismo primario y es el tipo de hipofunción tiroidea más frecuente. Se distinguen dos formas: hipotiroidismo subclínico e hipotiroidismo clínico (4).

La medición clínica de la Triyodotironina (T3), Tiroxina (T4), Hormona estimulante de Tiroides (TSH) y anti-TPO son métodos efectivos, para la determinación de estas alteraciones (5). Las concentraciones de TSH permiten diferenciar el hipotiroidismo primario del hipotiroidismo secundario. Los niveles FT3 y FT4 altos indican hipertiroidismo y los valores bajos hipotiroidismo. Los mismos son utilizados para valorar la función tiroidea especialmente cuando el paciente presenta alteraciones concurrentes (6).

El hipotiroidismo es frecuente a nivel mundial, es más prevalente en las mujeres especialmente a medida que se incrementa la edad (4 a 5 veces). En mujeres a partir de los 40 años, se han encontrado cifras del $8 \%$ al $10 \%$, afectando a más del $12 \%$ de las mujeres mayores de 60 años. Estos datos están relacionados con mayor susceptibilidad a alteraciones de la 
autoinmunidad, variaciones fisiológicas y hormonales del sistema endócrino en diferentes etapas de la vida $(7,8)$.

Según la Organización Mundial de la Salud, las patologías tiroideas tienen una prevalencia del $10 \%$ en la población mundial, pueden presentarse en distintas edades, en la infancia entre 4 a 5 años $(3,4$ $\%$ al $6 \%$ ), la tendencia aumenta con la edad, así en hombres y mujeres mayores de 70 años es del $20 \%(5,9)$. También, en Estados Unidos el hipo e hipertiroidismo tienen una prevalencia del 1,3\% en la población, siendo más evidente en el sexo femenino (10).

En Ecuador investigaciones recientes señalan que el hipotiroidismo se presenta cerca del $8 \%$ en la población adulta, y que el hipotiroidismo congénito tiene una incidencia desde 1 en 1.500 nacimientos (11).

El hipotiroidismo puede provocar disminución del metabolismo basal, los síntomas principales incluyen fatiga, afección de la frecuencia cardíaca, cansancio, hipersensibilidad al frío y, en mujeres se presentan alteraciones menstruales, constipación, sequedad de la piel y aumento de peso inexplicable que es más común en mujeres de edad avanzada (12).

Otro de los problemas asociados a esta patología es, el aumento del riesgo de desarrollar aterosclerosis y enfermedad cardiovascular, elevación de colesterol total y LDL-C con disminución de colesterol asociado a HDL-C, hipertensión arterial, inflamación con aumentos de proteína C reactiva ultrasensible, hiperinsulinemia, hipertrigliceridemia y síntomas neuropsiquiátricos $(13,14)$.

El hipotiroidismo constituye un motivo de consulta frecuente a nivel mundial y el Ecuador no escapa a esta problemática. Rodríguez y cols., (2016) señalan que en Ecuador la morbilidad de hipotiroidismo e hipertiroidismo se encuentra cercano al $8 \%$ en personas adultas. La tasa de prevalencia de hipotiroidismo congénito tiene una incidencia relativamente alta desde 1 en 1,500 nacimientos (15).

En la provincia de Santa Elena una gran cantidad de pacientes que acuden a consulta externa presentan signos de depresión, fatiga, obesidad, hiperlipidemias, y otros cuadros clínicos inespecíficos que sugieren la presencia de hipotiroidismo en la población. Sin embargo, no existe ningún estudio que indique cómo se encuentra esta problemática en esta zona del país.

El presente estudio, tiene como objetivo caracterizar el hipotiroidismo primario en pacientes que acuden al laboratorio clínico del Centro de Especialidades IESS de la Libertad, mediante la medición de niveles de FT3, FT4, TSH.

\section{MATERIALES Y MÉTODOS}

e realizó un estudio de tipo

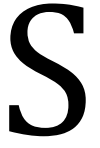
descriptivo, de diseño documental, retrospectivo. El universo muestral lo constituyen los datos de las historias clínicas de 2451 pacientes con sospecha de enfermedad tiroidea que acudieron al servicio de laboratorio del Centro de Especialidades IESS La Libertad durante el período enero 2018 a enero 2020, para lo cual se procedió a revisar archivos de historial de resultados de los pacientes atendidos en el laboratorio.

Se incluyeron pacientes de sexo masculino y femenino, pertenecientes a los grupos etarios establecidos por el MSP de Ecuador (niñez, adolescencia, adulto joven, adulto mediano, y adulto mayor) que cuentan con resultados de niveles séricos de FT3, FT4 y TSH. 
Se excluyeron a los pacientes que no contaban con información relacionada a niveles de FT3, FT4, TSH, pacientes con otro tipo de diagnóstico y las muestras que no cumplieron con los criterios de calidad establecidos por el laboratorio.

Para el procesamiento y análisis de la información se utilizó el paquete estadístico SPSS versión 21.0. Para el análisis de los resultados se empleó estadística descriptiva, análisis de frecuencias y chi-cuadrado. Para la presentación de los resultados se utilizaron tablas cruzadas y gráficos de barras.

La muestra se distribuyó por sexo y por grupos etarios $(0-9,10-19,20-44,45-$ $64,>64)$. Para realizar comparaciones, las edades se agruparon en población general, niñez, adolescencia, adulto joven, adulto mediano y adulto mayor.

\section{Procedimientos éticos}

La investigación siguió las normas éticas para la investigación con seres humanos, establecidas en la declaración de Helsinki, específicamente en sus adendum Declaración de Taiwan del año 2016, Declaración de la Asociación Médica Mundial (AMM), sobre las consideraciones éticas de las bases de datos de salud y biobancos. Se contó con la aprobación de los directores administrativo y médico del Centro de Especialidades IEES, la Libertad, en Ecuador para la utilización de los datos a a fines del desarrollo de la investigación. El uso de los datos derivará en beneficio total de la sociedad como fue planteado en la justificación de la investigación. En la información que se muestra en los resultados se protege la dignidad, autonomía, privacidad y confidencialidad de los participantes en la investigación en todo momento. Los datos fueron resguardados siendo usados exclusivamente por el investigador para los fines declarados en esta investigación. Los resultados presentados son totalmente anónimos y los datos estuvieron codificados, de modo que se asegure la privacidad del paciente. Los pacientes podrán retirar sus datos cuando lo deseen.

\section{RESULTADOS}

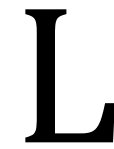
a muestra total constituye a la historia clínicas de 2451 pacientes que acudieron al servicio de laboratorio con sospecha de enfermedad tiroidea, los cuales cumplieron con los criterios de inclusión establecidos para este estudio.

Para determinar el número de pacientes con hipotiroidismo fueron valorados los niveles de TSH y FT4, con estos hallazgos de laboratorio se encontraron 636 pacientes que presentaron incremento de los niveles de TSH, de los cuales, 617 pacientes se los puede catalogar como hipotiroidismo primario, de estos, 76 pacientes presentaron hipotiroidismo clínico al presentar niveles de TSH incrementados y niveles de FT4 disminuidos, y 541 pacientes presentaron hipotiroidismo subclínico al mostrar niveles de TSH incrementados y FT4 dentro de los parámetros de normalidad.

Se descartaron 19 pacientes que presentan niveles incrementados de TSH y FT4, ya que puede estar relacionado a hipotiroidismo central o estar relacionado al uso de medicación.

En la Tabla 1, se indica la asociación entre niveles de TSH y FT4 en donde se estableció el número de pacientes con hipotiroidismo primario y su posterior clasificación como hipotiroidismo clínico y subclínico. 
Tabla 1. Asociación de niveles de TSH y FT4 para determinar tipos de hipotiroidismo.

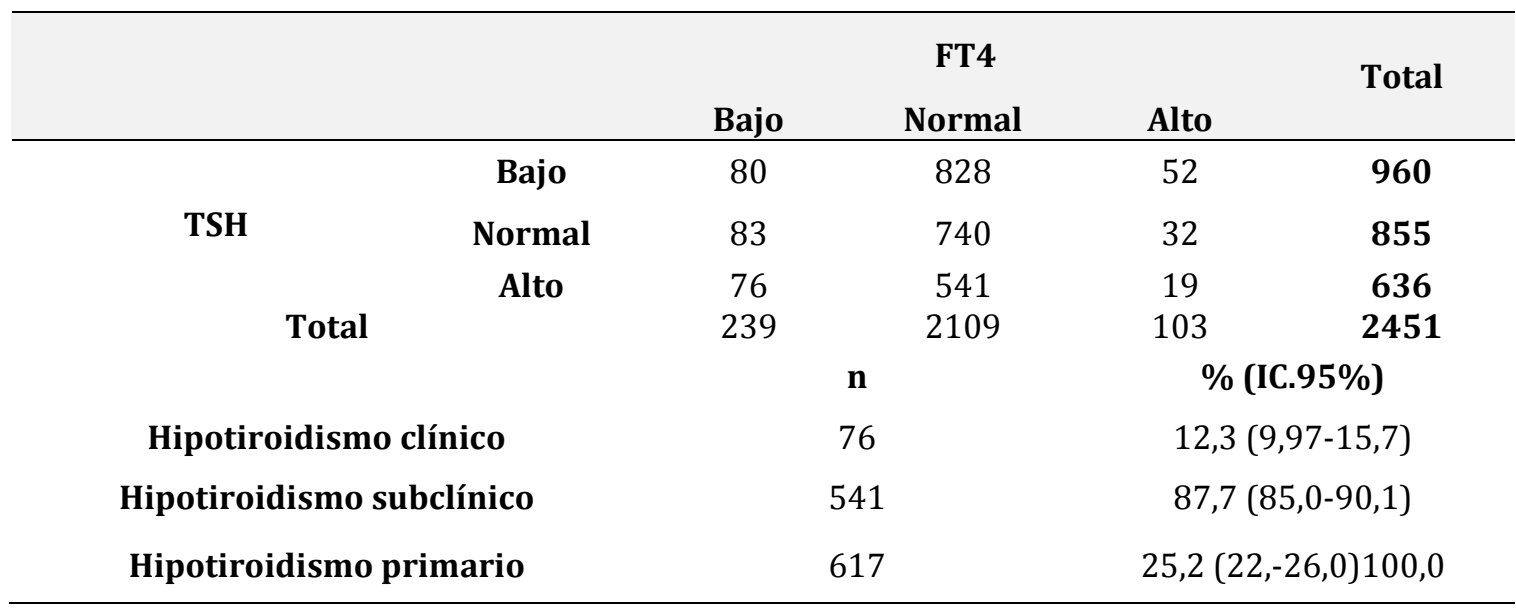

Al evaluar las historias de los 2451 pacientes se determinó que 617 pacientes cumplieron criterios de los diagnósticos de hipotiroidismo primario lo cual representa una prevalencia del $25,2 \%$ tal como se muestra en la Tabla 1.
En la Tabla 2, se observan los datos estadísticos correspondientes a casos de hipotiroidismo primario y su relación al sexo.

Tabla 2. Variables sociodemográficas en pacientes diagnosticados con hipotiroidismo primario según sexo, atendidos en el Centro de especialidades IEES La Libertad de Santa Elena periodo 2018 - 2020.

\begin{tabular}{|c|c|c|c|c|c|c|}
\hline & \multicolumn{2}{|c|}{$\begin{array}{l}\text { 1. Hipotiroidismo } \\
\text { clínico }\end{array}$} & \multicolumn{2}{|c|}{$\begin{array}{l}\text { 2. Hipotiroidismo } \\
\text { subclínico }\end{array}$} & \multicolumn{2}{|c|}{ 3. Total } \\
\hline & $\mathbf{n}$ & $\%$ & $\mathbf{n}$ & $\%$ & $\mathbf{n}$ & $\%$ - IC \\
\hline Femenino & 46 & 60,5 & 391 & 72,3 & 437 & $70,8(67,1-74,2)$ \\
\hline Masculino & 30 & 39,5 & 150 & 27,7 & 180 & $29,2(25,7-32,8)$ \\
\hline Total & 76 & 100 & 541 & 100 & 617 & 100 \\
\hline $\mathbf{X}^{2} ; \mathbf{p}$ & \multicolumn{6}{|c|}{$X^{2}=3,900 ; p=0,043$} \\
\hline
\end{tabular}

Los datos presentados en la tabla anterior destacan el tipo de hipotiroidismo primario que se asocia al sexo $\mathrm{X}^{2}=3,900$; $\mathrm{p}=0,043$. La prueba $\mathrm{Z}$ de comparación de proporciones $\quad(\mathrm{p}<0,050) \quad$ detectó diferencias significativas de las proporciones del tipo de hipotiroidismo según el sexo. Los hombres se clasifican mayoritariamente con hipotiroidismo clínico $(39,5$ vs 27,7$)$. Las mujeres tienen mayor frecuencia de hipotiroidismo subclínico $(72,3$ vs 60,5$)$.

En la Tabla 3, se muestran los valores estadísticos de Hipotiroidismo clínico y subclínico según el grupo etario. 
Tabla 3. Variables sociodemográficas en pacientes diagnosticados con hipotiroidismo primario evaluados según grupo etario, atendidos en el Centro de especialidades IEES La Libertad de Santa Elena periodo 2018-2020.

\begin{tabular}{ccccccc}
\hline & \multicolumn{2}{c}{$\begin{array}{c}\text { Hipotiroidismo } \\
\text { clínico }\end{array}$} & \multicolumn{2}{c}{ Hipotiroidismo } \\
subclínico & \multicolumn{2}{c}{ Total } \\
& $\mathbf{n}$ & $\mathbf{\%}$ & $\mathbf{n}$ & $\mathbf{\%}$ & $\mathbf{n}$ & $\mathbf{\%}$ \\
\hline Niñez & 1 & 1,3 & 12 & 2,2 & 13 & 2,1 \\
Adolescencia & 11 & 14,5 & 37 & 6,8 & 48 & 7,8 \\
Adulto joven & 16 & 21,1 & 134 & 24,8 & 150 & 24,3 \\
Adulto & 32 & 42,1 & 243 & 44,9 & 275 & 44,6 \\
Adulto mayor & 16 & 21,1 & 115 & 21,3 & 131 & 21,2 \\
Total & $\mathbf{7 6}$ & $\mathbf{1 0 0}$ & $\mathbf{5 4 1}$ & $\mathbf{1 0 0}$ & $\mathbf{6 1 7}$ & $\mathbf{1 0 0}$ \\
\hline $\mathbf{X}^{2} ; \mathbf{p}$ & \multicolumn{6}{c}{$\mathbf{X 2}=5,748 ; \mathrm{p}=0,219$} \\
\hline
\end{tabular}

Se puede observar que el hipotiroidismo clínico en pacientes adultos se presenta en el orden del $42,1 \%$ $(\mathrm{N}=32)$, a estos le siguen los adultos mayores y adultos jóvenes con datos iguales $(16 / 21,1 \%)$, seguido de adolescentes y niños que representan el $14,5 \%$ y 1,3 \% respectivamente. En el caso del hipotiroidismo subclínico, se

puede apreciar que en la edad adulta se presenta en 243 pacientes $(44,9 \%)$, seguido de los adultos jóvenes $(134 / 24,8$ $\%)$, adultos mayores $(115 / 21,3 \%)$ adolescentes $6,8 \%$ y niños $2,2 \%$ tal como lo lo destaca la Tabla 3.

Seguidamente, se muestra la Tabla 3A sobre la tabla cruzada tipo de hipotiroidismo del grupo etario.

Tabla 3-A. Tabla cruzada tipo de hipotiroidismo grupo etario.

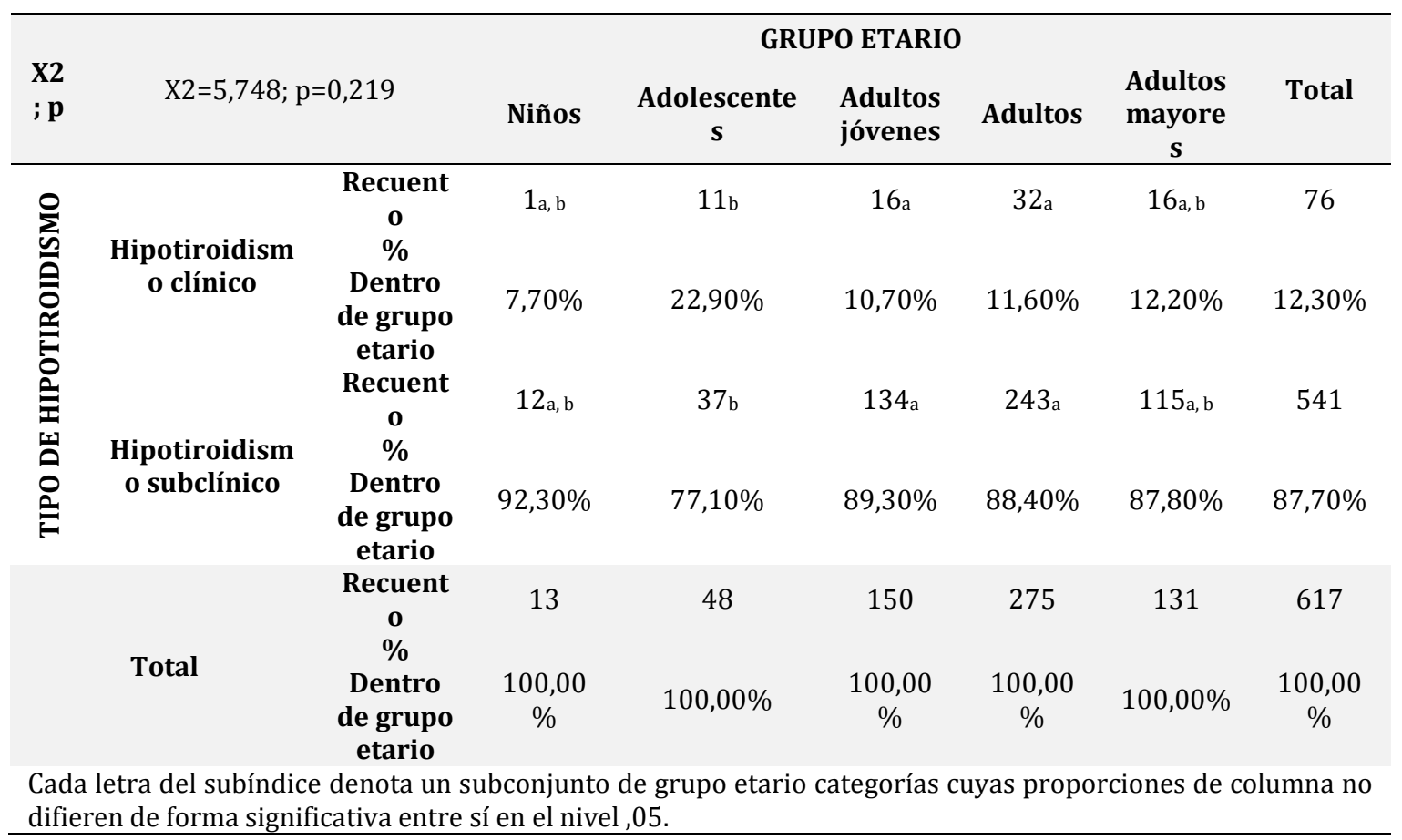


La Tabla 3-A, explica el adolescentes, igual adulto joven y adulto, comportamiento para cada tipo de igual en niños y adultos mayores por ese hipotiroidismo según el sexo, El orden.

hipotiroidismo clínico es igual en niños y

En la Tabla 4, se muestra a continuación adultos mayores; adulto joven y adulto $\mathrm{y}$ mayor en adolescentes por ese orden. El hipotiroidismo subclínico es menor en los datos estadísticos que muestran la frecuencia de pacientes hipotiroideos y comorbilidades relacionadas.

Tabla 4. Frecuencia de pacientes hipotiroideos y comorbilidades en pacientes atendidos en el Centro de especialidades IEES La Libertad de Santa Elena periodo 2018 - 2020.

\begin{tabular}{|c|c|c|c|c|c|c|c|}
\hline & & \multicolumn{2}{|c|}{$\begin{array}{l}\text { Hipotiroidismo } \\
\text { clínico }\end{array}$} & \multicolumn{2}{|c|}{$\begin{array}{l}\text { Hipotiroidismo } \\
\text { subclínico }\end{array}$} & \multicolumn{2}{|c|}{ Total } \\
\hline & & $\mathbf{n}$ & $\%$ & $\mathbf{n}$ & $\%$ & $\mathbf{n}$ & $\%$ \\
\hline \multirow{13}{*}{ 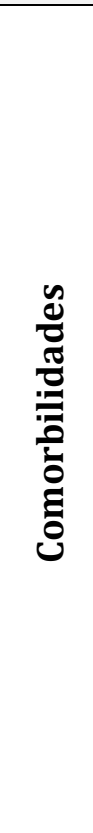 } & Ansiedad & 6 & 7,9 & 52 & 9,61 & 58 & 9,4 \\
\hline & Anemia & 5 & 6,6 & 18 & 3,33 & 23 & 3,7 \\
\hline & Artritis reumatoide & 0 & 0 & 6 & 1,11 & 6 & 1 \\
\hline & Diabetes Mellitus & 14 & 18,4 & 73 & 13,49 & 87 & 14,1 \\
\hline & Depresión & 1 & 1,3 & 18 & 3,33 & 19 & 3,1 \\
\hline & Dislipidemia & 14 & 18,4 & 145 & 26,8 & 159 & 25,8 \\
\hline & Embarazo & 0 & 0 & 4 & 0,74 & 4 & 0,6 \\
\hline & Hipertensión arterial & 29 & 38,2 & 187 & 34,57 & 216 & 35 \\
\hline & Infertilidad & 0 & 0 & 4 & 0,74 & 4 & 0,6 \\
\hline & Obesidad & 3 & 3,9 & 20 & 3,7 & 23 & 3,7 \\
\hline & Síndrome Metabólico & 4 & 5,3 & 9 & 1,66 & 13 & 2,1 \\
\hline & Desnutrición & 0 & 0 & 5 & 0,92 & 5 & 0,8 \\
\hline & Total & 76 & 100 & 541 & 100 & 617 & 100 \\
\hline$X^{2} ; p$ & $X 2=12,963 ; p=0,296$ & & & & & & \\
\hline
\end{tabular}

En la evaluación de comorbilidad se aprecia que las asociadas tanto al hipotiroidismo clínico y subclínico son en orden de frecuencia: hipertensión arterial (35\%), dislipidemia $(25,8 \%)$ y diabetes mellitus (14,1\%). (Tabla 4).
En la Tabla 5, se muestra la relación entre comorbilidades y edad en pacientes con hipotiroidismo. 
Tabla 5. Relación de comorbilidades y edad en pacientes hipotiroideos atendidos en el Centro de especialidades IEES La Libertad de Santa Elena periodo 2018 - 2020

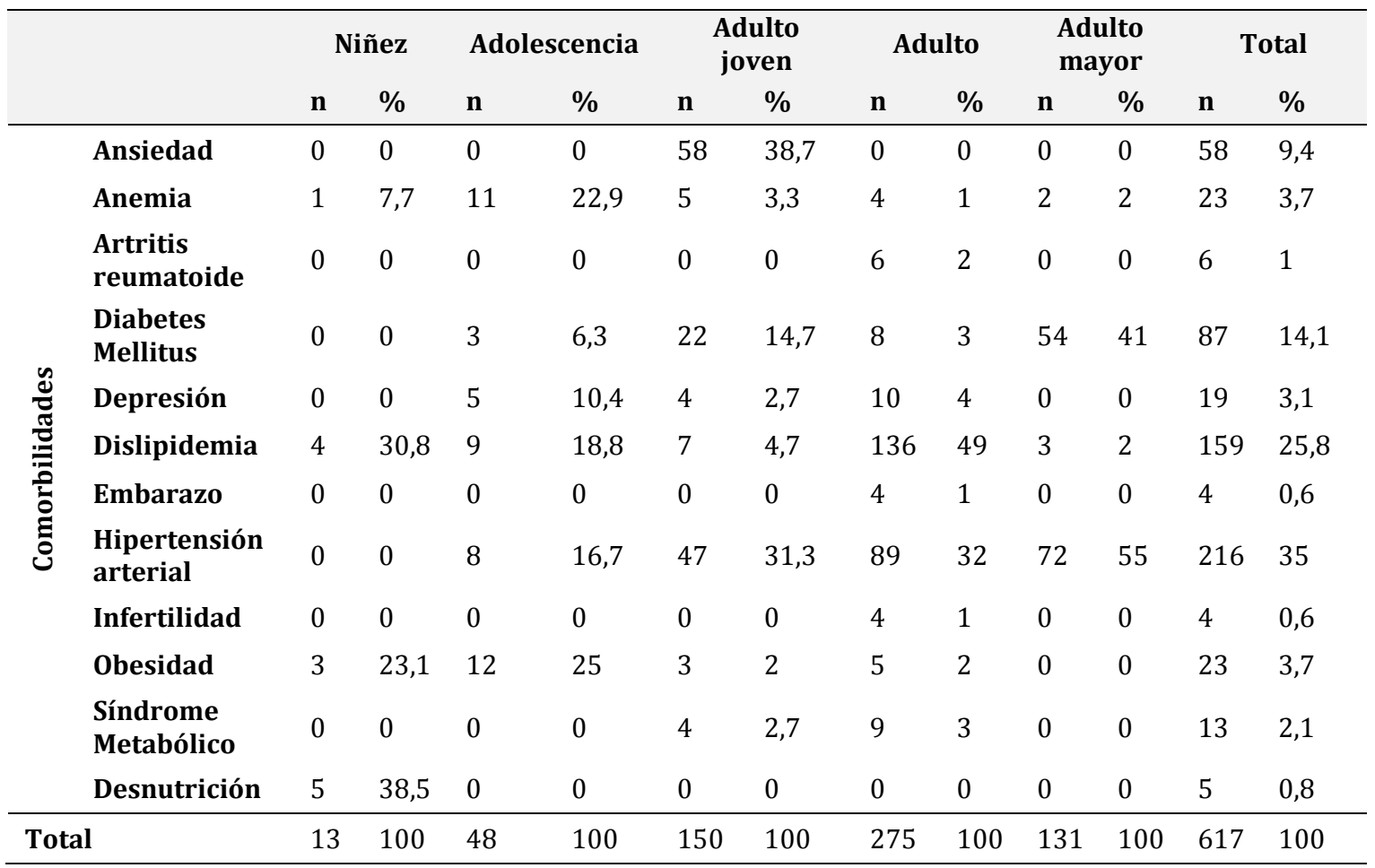

Con respecto a las comorbilidades, es importante mencionar que estas entidades pueden ser concomitantes o ser secundarias al hipotiroidismo ya que, al disminuir el metabolismo basal, los procesos metabólicos enlentecen y pueden generar dichas patologías.

En la muestra general, el $35 \%$ de pacientes presentan hipertensión arterial, siendo más frecuente entre los adultos mayores $(\mathrm{N}=72)$ y adultos $(\mathrm{N}=89)$, de igual manera el 25,8 \% de pacientes presentan dislipidemias observándose que la población de adultos representa la población con mayor número de pacientes con esta patología $(\mathrm{N}=136)$ y por último el $14,1 \%$ de pacientes presentan diabetes mellitus, siendo más frecuentes entre los adultos mayores $(\mathrm{N}=54)$

\section{DISCUSIÓN}

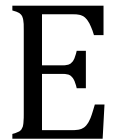
l hipotiroidismo es un síndrome clínico resultante de la insuficiencia de hormona tiroidea libre circulante y en tejidos periféricos, que conlleva a la disminución generalizada de procesos metabólicos con consecuencias graves en el desarrollo físico y mental (16).

Los desequilibrios hormonales son estimados como causas que desencadenan enfermedades crónicas como la diabetes, hipertensión y las alteraciones tiroideas. Según Rivera-Hernández et al., alrededor del $7 \%$ de la población padece algún tipo de enfermedad tiroidea, esta aumenta a casi un $12 \%$ en la población con diabetes tipo II (17). Por otro lado, según datos de 
la Organización Mundial de la Salud, las patologías tiroideas tienen una prevalencia del $10 \%$ y va en aumento en personas mayores de 70 años (18).

En este contexto, dentro del presente estudio los datos estadísticos muestran, una prevalencia de hipotiroidismo de 25,2 $\%$ cifras elevadas en relación con la literatura mundial, así mismo el estudio indica que el hipotiroidismo de tipo subclínico es el más frecuente $(87,7 \%)$, mientras que el hipotiroidismo clínico, se encuentra en el orden del 12,3\%.

Dentro de la revisión bibliográfica se encontró que en la investigación realizada por Talwalkar y cols., en India en el año 2019, los investigadores indican que en pacientes con diabetes mellitus tipo II, hipertensión arterial y pacientes con diabetes más hipertensión, la prevalencia de hipotiroidismo fue del 24,8 \%, 33,5 \% y $28,9 \%$ respectivamente, cifras similares a la presente investigación. En relación con las cifras de hipotiroidismo clínico y subclínico los datos del estudio de Talwalkar indican que el más frecuente es el de tipo clínico 77,3 \% y el 22,7 \% hipotiroidismo subclínico (19). Estas cifras son diferentes a la de este estudio en donde el tipo subclínico es más frecuente.

En contraste a estos datos un estudio realizado en Colombia, se indica que se documentó un $22 \%$ de disfunción tiroidea, principalmente relacionada con hipotiroidismo subclínico, de los cuales el $11 \%$ eran de sexo masculino y el $1 \%$ de sexo femenino, además se indicó que una paciente tenía hipotiroidismo clínico (1\%) (20), estos estudios se realizaron en pacientes considerados como adultos mayores, según los autores, estos hallazgos se deben a que la prevalencia encontrada es considerablemente más elevada que la reportada en otras publicaciones, ya que los pacientes del estudio pertenecían a un centro de referencia de pacientes con diferentes patologías, entre ellas la disfunción tiroidea.

Al igual que los autores del estudio en India, se cree que el desarrollo de pruebas diagnósticas más sensibles para la detección de TSH podría incidir en la detección de una alta prevalencia de hipotiroidismo, si bien este estudio no tiene como objetivo hacer una relación directa de hipotiroidismo diabetes e hipertensión, la investigación indica que existe una alta frecuencia de pacientes con hipertensión arterial ,dislipidemias y diabetes mellitus tipo II lo que incidiría en la prevalencia obtenida. Además, la alta población de adultos y adultos mayores de este estudio podrían contribuir a la elevada prevalencia encontrada.

En cuanto a la edad, como se mencionó anteriormente, en esta investigación el hipotiroidismo subclínico, se presenta con mayor frecuencia en el grupo etario correspondiente a adultos, adultos jóvenes $\mathrm{y}$ adultos mayores en orden de frecuencias lo que incurriría en la prevalencia elevada.

Se han reportado también en otros estudios prevalencias altas de hipotiroidismo, así en Chile, durante la Encuesta Nacional de Salud 2009-2010 se evaluó la prevalencia de disfunción tiroidea en personas de 15 o más años de edad y se encontró que la prevalencia de hipotiroidismo en ambos sexos fue de 19.4\%; en hombres tuvo una prevalencia de $17.3 \%$ y en mujeres $21.5 \%$ (21).

Esta alta prevalencia según los investigadores indica, se puede deber a que en la muestra se incluyó un alto porcentaje de adultos mayores los que estadísticamente presentan mayor frecuencia de hipotiroidismo.

Comparando los resultados de la literatura internacional y nacional en relación a la frecuencia de hipotiroidismo y sexo de los pacientes, Escobar y cols., 
señalan que la prevalencia de hipotiroidismo es dos veces más frecuente en mujeres $(4,8 \%$ a $10 \%)$ que en hombres $(0,9 \%)$ y en función de la edad, con una prevalencia de $20 \%$ y $16 \%$ en mujeres y hombres respectivamente mayores de 60 años (22). Según el presente estudio, los datos estadísticos mostrados permiten apreciar que, en la población analizada con diagnóstico de hipotiroidismo (clínico y subclínico), es mayor en mujeres que en hombres.

Datos similares a esta investigación reporta Guevara-Sánchez en Costa Rica, quien indica que se halló una prevalencia de $11,8 \%$ en pacientes con hipotiroidismo subclínico. Este cuadro subclínico fue 3 veces más frecuente en mujeres que en hombres y constituyó la mayoría de los casos de disfunción tiroidea encontrados, especialmente en pacientes mayores de 55 años (10).

En cuanto a estudios nacionales, en la investigación realizada por Cando y cols., en Ecuador, se muestra que las alteraciones tiroideas en la población de estudio se presentaron en un 78,6\% en mujeres y en $21,4 \%$ en hombres. Se encontraron 4 casos de hipotiroidismo subclínico 3 de ellos en el sexo femenino y un caso en el masculino (9)

La literatura indica que esta condición generalmente aparece debido a que, durante su ciclo de vida, las mujeres manifiestan cambios hormonales, fisiológicos y trastornos autoinmunitarios que influyen en el funcionamiento normal de la glándula tiroides.

Según Sichieri y cols., en Latinoamérica la prevalencia de la disfunción tiroidea varía según la edad, la raza y el sexo, siendo más frecuente en las mujeres que en los hombres, en los blancos y mulatos más que en los negros. Se evidencian cifras de hasta $10 \%$ de hipotiroidismo y 2,2 \% de hipertiroidismo. La prevalencia de hipertiroidismo subclínico es 3,9\% y la de hipotiroidismo subclínico 11,8 \% (23).

En la investigación sobre Hipotiroidismo por Leng y cols., en la cual realizan un análisis sobre varios estudios ejecutados a nivel mundial indican que en Holanda en pacientes de ambos sexos con un rango de edad superior a 45 años se reporta una prevalencia de 0.8 a 9,1 \% en pacientes con hipotiroidismo subclínico, así mismo en una investigación realizada en 1373 pacientes de ambos sexos con una edad superior a 65 años se encontró una prevalencia de hipotiroidismo subclínico de 5,7- 6,5 \%, en EEUU se halló una prevalencia de hipotiroidismo subclínico entre 2,5 a $7,9 \%$ en pacientes de ambos sexos con una edad mayor a 60 años (24) Estas cifras son relativamente más bajas a nuestro estudio y la diferencia se cree está basada en el alto monitoreo y seguimiento de estos pacientes que cuentan con sistemas de salud más robustos, la bibliografía señala que la amplia variación entre los distintos estudios probablemente reflejan la naturaleza dispar de las poblaciones, siendo evaluados con respecto a su género, ingesta de yodo, grupos de edad, grupos raciales $y$ predominio de enfermedad tiroidea tratada (25).

Por otro lado, es importante investigar la procedencia de los pacientes, ya que en las zonas rurales existen condiciones sociales y ambientales diferentes, estudios científicos indican que factores como un aumento en el uso no regulado de plaguicidas, exposición a contaminantes industriales, incluidos los bociógenos, y consumo de agua no potable en la última década puede servir como otras causas de hipotiroidismo (26). 
Al realizar un análisis de las comorbilidades que presentan los pacientes hipotiroideos motivo de este estudio, el $35 \%$ tienen hipertensión arterial, el 25,8 \% presentan dislipidemias y el $14,1 \%$ tienen diabetes mellitus, siendo más frecuentes entre los adultos y adultos mayores. Los resultados obtenidos están en relación con la edad de los pacientes del estudio que como ya se mención anteriormente la población de adultos y adultos mayores es más numerosa.

Los hallazgos de esta investigación son análogos a la investigación de Frías López y cols., quienes reportan un porcentaje del $23 \%$ de sujetos con hipertensión, los autores indican que esta menor prevalencia puede ser debida a que un 23 $\%$ de sujetos tienen edades inferiores a 35 años y casi un $55 \%$ de sujetos son menores de 50 años. Informan además que la Diabetes Mellitus tipo II, está presente en el $13 \%$ de los sujetos con hipotiroidismo y un $40 \%$ de sujetos presentan dislipemia (27).

Por otro lado, datos publicados por Banegas y cols., reflejan que la población general española presenta una prevalencia de casi un $40 \%$ de pacientes hipotiroideos con hipertensión arterial en edades medias y un $60 \%$ en mayores de 60 años (28).

Gordillo y cols., en su trabajo sobre la influencia de enfermedades tiroideas en el desarrollo de enfermedad cardiovascular indican que la alteración en el perfil lipídico (hipercolesterolemia e hipertrigliceridemia) representó el segundo parámetro más alto en adultos mayores, en la población hipotiroidea con un $62,50 \%$ y $53,13 \%$ respectivamente (29).

\section{CONCLUSIONES}

$\mathrm{E}$ l estudio indica que existe una alta frecuencia de hipotiroidismo primario en la población que concurre al control de salud. Los datos demuestran que los hombres se clasifican mayoritariamente con hipotiroidismo clínico y que en el grupo de las mujeres se presenta con mayor frecuencia el hipotiroidismo subclínico.

El hipotiroidismo clínico se presenta en mayor número en pacientes adultos, seguido de los adultos mayores y adultos jóvenes. De igual manera, en el caso del hipotiroidismo subclínico, se presenta en orden de frecuencia en la edad adulta, adultos jóvenes y en adultos mayores lo que concuerda con la literatura. Los niveles elevados de hipotiroidismo primario pueden deberse a que la muestra lo conforman un alto número de pacientes de adultos y adultos mayores en los cuales se presenta con mayor frecuencia esta patología.

La presencia de comorbilidades como hipertensión arterial, dislipidemias $\mathrm{y}$ diabetes mellitus tipo II están presentes en los pacientes hipotiroideos estudiados, por lo que se deberían hacer estudios que relacionen estas patologías con hipotiroidismo.

Es importante fomentar la frecuencia de ejecución de pruebas que establezcan la concentración sérica de la hormona estimulante de la tiroides TSH y la T4, con la finalidad de hacer un diagnóstico precoz y tratamiento oportuno para así evitar que los pacientes puedan progresar a un hipotiroidismo clínico o franco. 


\section{REFERENCIAS BIBLIOGRÁFICAS}

1. Fundación Merck. Manual de Patología Tiroidea. Fundación Merck Salud.

2. Pulgarín SR, Sánchez LMM, Jaramillo LIJ. Enfermedad tiroidea: Una aproximación clínica y genética. Archivos de Medicina (Col). 16(2):359-72.

3. Parlá Sardiñas J. Hipotiroidismo. Revista Cubana de Endocrinología. 2012;23(3):208-12.

4. IMSS. Diagnóstico y tratamiento de hipotiroidismo primario y subclinico en el adulto | Hipotiroidismo | Tiroides. Scribd.

5. Leiva ette, Morales J, Villacis S, Quishpe

G. Hipotiroidismo, enfoque actual. |.

6. Pagana $K$, Pagana $T$, Pagana $T$. Guía de pruebas diagnósticas y de laboratorio 13th Edition.

7. Belén LR, Maffei L, Alorda B, Squillace C, Rossi ML, Oliva ML, et al. Prevalencia de hipotiroidismo y su asociación con factores de riesgo cardiometabólicos en mujeres adultas argentinas. Revista Española de Nutrición Humana y Dietética. 2015;19(3):146-52.

8. Iwen KA, Schröder E, Brabant G. Thyroid Hormones and the Metabolic Syndrome. Eur Thyroid J. junio de 2013;2(2):83-92.

9. Cando-Brito VM, Rodríguez-Vinueza VI, Escobar-Arrieta SN, Toaquiza-Aguagallo NC. Determinación de alteraciones tiroideas en pacientes voluntarios, de un dispensario de salud ocupacional, Riobamba-Ecuador. Polo del Conocimiento. 2020;5(5):465-83.

10. Guevara Sánchez 0 , Holst Schumacher I, Boza Oreamuno S, Barrantes Santamaría M, Chinchilla Monge R, Alvarado Ulate P. Disfunción tiroidea subclínica en población adulta costarricense. An Fac med. 9 de enero de 2016;76(4):333.

11. Rodríguez Ramos JF, Boffill Corrales AM, Rodríguez Soria A. Factores de riesgo de las enfermedades tiroideas. Hospital del Seguro Social Ambato. Revista de Ciencias
Médicas de Pinar del Río. 2016;20(5):11328.

12. Jara Albarràn A. Endocrinologia. Editorial medica España 2011.

13. Meier C, Staub J-J, Roth C-B, Guglielmetti M, Kunz M, Miserez AR, et al. TSH-Controlled l-Thyroxine Therapy Reduces Cholesterol Levels and Clinical Symptoms in Subclinical Hypothyroidism: A Double Blind, Placebo-Controlled Trial (Basel Thyroid Study). The Journal of Clinical Endocrinology \& Metabolism. 2001;86(10):4860-6.

14. Kvetny J, Heldgaard PE, Bladbjerg EM, Gram J. Subclinical hypothyroidism is associated with a low-grade inflammation, increased triglyceride levels and predicts cardiovascular disease in males below 50 years. Clin Endocrinol (Oxf). agosto de 2004;61(2):232-8.

15. Rodríguez Ramos JF, Boffill Corrales AM, Rodríguez Soria A. Factores de riesgo de las enfermedades tiroideas. Hospital del Seguro Social Ambato. Revista de Ciencias Médicas de Pinar del Río. 2016;20(5):11328.

16. Hospital General de Niños Pedro de Elizalde. Hipotiroidismo. Criterios de diagnóstico

y tratamiento en Pediatría. Segunda Edición. Buenos Aires: Ediciones Journal, 2012.

17. Rivera-Hernández A, HuertaMartínez H, Centeno-Navarrete Y, ZuritaCruz JN. Actualización en hipotiroidismo congénito: etiología, cuadro clínico, diagnóstico y tratamiento. Segunda parte. :7.

18. Alexander EK, Pearce EN, Brent GA, Brown RS, Chen H, Dosiou C, et al. 2017 Guidelines of the American Thyroid Association for the Diagnosis and Management of Thyroid Disease During Pregnancy and the Postpartum. Thyroid. 2017;27(3):315-89.

19. Talwalkar P, Deshmukh V, Bhole M. Prevalence of hypothyroidism in patients with type 2 diabetes mellitus and 
hypertension in India: a cross-sectional observational study. Diabetes Metab Syndr Obes. 2019;12:369-76.

20. Chaves W, Amador D, Tovar $H$. Prevalencia de la disfunción tiroidea en la población adulta mayor de consulta externa. 2018;43:7.

21. Liberman G. Prevalencia e incidencia de los principales trastornos endocrinos y metabólicos. Revista Médica Clínica Las Condes. 1 de septiembre de 2013;24:73541.

22. Escobar M, Villamil M, Ruiz 0 . Prevalencia de anticuerpos antiperoxidasa y antitiroglobulina en jóvenes con hipotiroidismo subclínico y clínico. 2011;7.

23. Sichieri R, Baima J, Marante $T$, Vasconcellos MTLD, Moura AS, Vaisman M. Low prevalence of hypothyroidism among black and Mulatto people in a populationbased study of Brazilian women. Clinical Endocrinology. 2007;66(6):803-7.

24. Leng O, Razvi S. Hypothyroidism in the older population. Thyroid Research. 2019;12(1):2.

25. van den Beld AW, Visser TJ, Feelders RA, Grobbee DE, Lamberts SWJ. Thyroid Hormone Concentrations, Disease, Physical Function, and Mortality in Elderly Men.
The Journal of Clinical Endocrinology \& Metabolism. 1 de diciembre de 2005;90(12):6403-9.

26. Bagcchi S. Hypothyroidism in India: more to be done. The Lancet Diabetes \& Endocrinology. octubre de 2014;2(10):778.

27. Frías López $\mathrm{M}^{a} \mathrm{C}$, Tárraga López PJ, Rodríguez Montes JA, Solera Albero J, Celada Rodríguez Á, López Cara MA, et al. Hipotiroidismo subclínico y factores de riesgo cardiovascular. Nutrición Hospitalaria. 2011;26(6):1355-62.

28. Banegas JR, Villar F, Graciani A, Rodríguez-Artalejo F. Epidemiología de las enfermedades cardiovasculares en España. Revista Española de Cardiología Suplementos. 2006;6(7):3G-12G.

29. Iñiguez AMG, Palacios DRM. Influencia del hipotiroidismo e hipertiroidismo en el desarrollo de enfermedad cardiovascular. Revista Médica-Científica CAMbios HECAM. 2019;18(2):13-7.

Conflicto de intereses. La autora no declara ningún conflicto de intereses.

Fuente de financiamiento. La autora declara. Agradecimientos. La autora no declara. 